\title{
Nuevas perspectivas moleculares para el tratamiento de los meningiomas anaplásicos recurrentes: a propósito de un caso
}

\author{
Novel molecular perspectives for the treatment of recurrent anaplastic meningiomas: \\ a case report
}

\author{
- José M. Behaine ${ }^{1,2}$, Juan Armando Mejía, ${ }^{1,2}$, Nicolás Useche ${ }^{1,3}$, Sonia Bermúdez', ${ }^{1,3}$, Andrés Felipe Cardona $a^{1,4,5}$ \\ 'Instituto de Neurociencias, Universidad El Bosque (Bogotá, Colombia). \\ 2 Departamento de Neurocirugía, Fundación Santa Fe de Bogotá (Bogotá, Colombia). \\ Departamento de Imágenes Diagnósticas, Sección Neurorradiología, Fundación Santa Fe de Bogotá (Bogotá, Colombia). \\ " Grupo de Oncología Clínica y Traslacional, Instituto de Oncología, Fundación Santa Fe de Bogotá (Bogotá, Colombia). \\ ${ }^{5}$ Fundación para la Investigación Clínica y Molecular Aplicada al Cáncer (FICMAC); Investigador asociado ONCOLGroup (Bogotá, Colombia).
}

\begin{abstract}
Resumen
Se reporta el caso de una mujer de 53 años con un cuadro clínico de corta evolución consistente en cambios comportamentales que se asoció con alteración del afecto y de la atención, por lo que se diagnosticó un meningioma de alto grado frontal izquierdo, que requirió manejo quirúrgico en tres oportunidades, radioterapia y dos líneas de tratamiento médico (hidroxiurea e imatinib) sin respuesta; ante la evidente progresión de la enfermedad, se decidió administrar octreotide más everolimus, intervención dirigida contra la expresión anormal de receptores de somatostatina tipo 2 y la disfunción molecular de la vía de señalización PIP3K/mTOR/AKT. Con dicha intervención, se alcanzó respuesta parcial.
\end{abstract}

Palabras clave: Meningioma, anaplasia, inhibidor mTOR, everolimus, receptor de somatostatina, octreotide.

\begin{abstract}
A 53-year-old woman who presented behavioral changes with attention and affection disturbances due to the diagnosis of a high grade meningioma requiring multiple surgeries followed by radiotherapy, hydroxyurea and imatinib; thereafter, the lesion progressed considering the use of octreotide plus everolimus to modulate the abnormal somatostatin receptor expression as well as the altered transcription by PIP3K/mTOR/AKT pathway. With this treatment the patient obtained a durable partial reponse.
\end{abstract}

Key words: Meningioma, anaplasia, mTOR inhibitor, everolimus, somatostatine receptor, octreotide.

\section{Introducción}

Los meningiomas son los tumores primarios más frecuentes del sistema nervioso central, y representan entre el 15 y $25 \%$ de las neoplasias de este origen. Su incidencia es de 6 por 100.000 habitantes/año'. La mayoría de los pacientes que presentan meningiomas son asintomáticos, por lo que buena parte de las lesiones representa un diagnóstico incidental durante la adultez ${ }^{1,2}$. Solo una minoría de estos tumores se clasifican como malignos, escenario que implica la presencia de anaplasia, y una posibilidad de recurrencia tras la exéresis quirúrgica entre el 50 y $80 \%$ a dos años ${ }^{1,2}$.
La mortalidad a cinco años para los tumores de mayor gradación excede el 80\%. La tabla 1 presenta la definición histológica de los meningiomas atípicos y anaplásicos, y sus principales desenlaces. El pilar del tratamiento para los meningiomas es la resección quirúrgica completa, no obstante, los tumores grado II y III tienen una supervivencia libre de progresión (SLP) entre el 40 y $85 \%$ a cinco años y una supervivencia global (SG) en el mismo período del 32 y $76 \%$, respectivamente ${ }^{1,2}$. Debido a su agresividad biológica, la radioterapia adyuvante constituye una herramienta complementaria en presencia de enfermedad residual 
Tabla 1. Características histopatológicas de los meningiomas atípicos y anaplásicos según la clasificación de la Organización Mundial de la Salud (2010)

\begin{tabular}{|c|c|c|c|}
\hline Características histológicas & $\begin{array}{l}\text { Porcentaje de } \\
\text { presentación }\end{array}$ & $\begin{array}{l}\text { Porcentaje } \\
\text { de recurrencia }\end{array}$ & SLP seis meses \\
\hline $\begin{array}{l}\text { Meningiomas atí́picos } \\
\geq 4 \text { mitosis/10 CAP }\left(\geq 2,5 / \mathrm{mm}^{2}\right) \text { o alguna de las siguientes características: } \\
\text { 1) Nucléolo prominente. } \\
\text { 2) Hipercelularidad }\left(\geq 53 \text { núcleos } / C A P ; \geq 118 / \mathrm{mm}^{2}\right) \text {. } \\
\text { 3) Invasión del cerebro. } \\
\text { 4) Presencia de necrosis. } \\
\text { 5) Formación de células pequeñas. } \\
\text { 6) Arquitectura de revestimiento. }\end{array}$ & $4-7 \%$ & $40 \%$ & $25 \%$ \\
\hline $\begin{array}{l}\text { Meningiomas anaplásicos } \\
\geq 20 \text { mitosis/10 CAP }\left(\geq 12,5 \mathrm{~mm}^{2}\right) \text { o alguna de las siguientes características: } \\
\text { 1) Pérdida difusa o focal de la apariencia meningotelial, resultando en carcinoma, sarcoma } \\
\text { o melanoma. }\end{array}$ & $1-2 \%$ & $50-80 \%$ & $<25 \%$ \\
\hline
\end{tabular}

o afectación cerebral'. En los tumores más agresivos, la cirugía y la radioterapia difícilmente logran un control adecuado de la enfermedad a largo plazo; por esto, se han investigado múltiples agentes quimioterapéuticos sin éxito. Debido a los resultados desalentadores con las intervenciones médicas clásicas, la atención se ha tornado progresivamente hacia los blancos moleculares $^{2}$, incluyendo el receptor para el factor de crecimiento derivado de las plaquetas (PDGFR) y las vías dependientes de mTOR $^{3}$.

La alteración genética más frecuente de los meningiomas es la monosomía del cromosoma 22, que se asocia en un $50 \%$ de los casos con pérdida de la banda 22q12. El principal gen localizado en esta región es el NF2 (neurofibromatosis tipo 2 o Merlin), en el que se han identificado mutaciones en todos los meningiomas derivados de la neurodermatosis y en el $50 \%$ de los esporádicos ${ }^{4}$. Sin embargo, la genómica de los meningiomas no relacionados con NF2 parecía desconocida hasta hace poco; recientemente, Brastianus y colaboradores presentaron la secuenciación exómica completa de 18 meningiomas de alto grado 5 , $y$, en paralelo, un segundo grupo de la Universidad de Yale evaluó extensamente el genotipo de 50 lesiones ${ }^{6}$. Ambos trabajos reportaron resultados similares, coincidiendo en que estos tumores tienen secuencias menos complejas dependientes de mutaciones no sinónimas que afectan los cromosomas 1, 7, 14, 19 y 22. Los genes alterados con mayor frecuencia fueron el NF2, TRFA7, KLF4, AKT1 y SMO. Un 79\% presentó mutaciones en alguno de estos cinco genes relacionados con vías de señalización dependientes de Hedgehog (Hh) y PI3k-AKT-mTOR. Esta última línea ya se había encontrado alterada con anterioridad en otros meningiomas atípicos y anaplásicos ${ }^{5}$ Por otra parte, aproximadamente el $90 \%$ de los meningiomas atípicos y anaplásicos sobreexpresan receptores de somatostatina tipo 2 (RS2), molécula relacionada con la angiogénesis y proliferación tumoral ${ }^{3,7,8}$.

A continuación, se expone el caso de una mujer de edad media con un meningioma anaplásico recurrente tratado en múltiples oportunidades con cirugía, imatinib e hidroxiurea, sin respuesta. Después de documentar una alta expresión de receptores de RS2, se inició everolimus más octeotride LAR, logrando una óptima respuesta.

\section{Descripción del caso}

Se trata de una mujer de 53 años sin antecedentes relevantes que presentó un cuadro de breve evolución caracterizado por cambios de la personalidad asociados a hipoprosexia y depresión. En el primer cuarto del 2011, se le diagnosticó una lesión extraaxial frontal izquierda con base de implantación en la hoz del cerebro. Fue llevada a cirugía obteniendo una patología que describió la presencia de un meningioma atípico con enfermedad residual, motivo por el que recibió radioterapia (60-200 cGy/día) sin complicaciones. Luego de esto, continuó en observación con estabilización de la enfermedad hasta 18 meses después, cuando se documentó recidiva a nivel frontal izquierdo, nuevamente con afectación de la hoz del cerebro e importante edema vasogénico más desviación de la línea media. Las dimensiones de los tumores fueron de $41 \times 29 \times 25 \mathrm{~mm}$ y se encontró una nueva lesión hacia el planum esfenoidal con extensión a la región frontoorbitaria del lado izquierdo (40 x 30 x $27 \mathrm{~mm}$ ). La figura 1 muestra las imágenes en las secuencias T1 con gadolinio. Dicho hallazgo condicionó un rápido deterioro funcional, por lo que se decidió realizar embolización selectiva de las lesiones y una nueva resección quirúrgica. La nueva patología encontró marcado pleomorfismo, sin pérdida del patrón de 


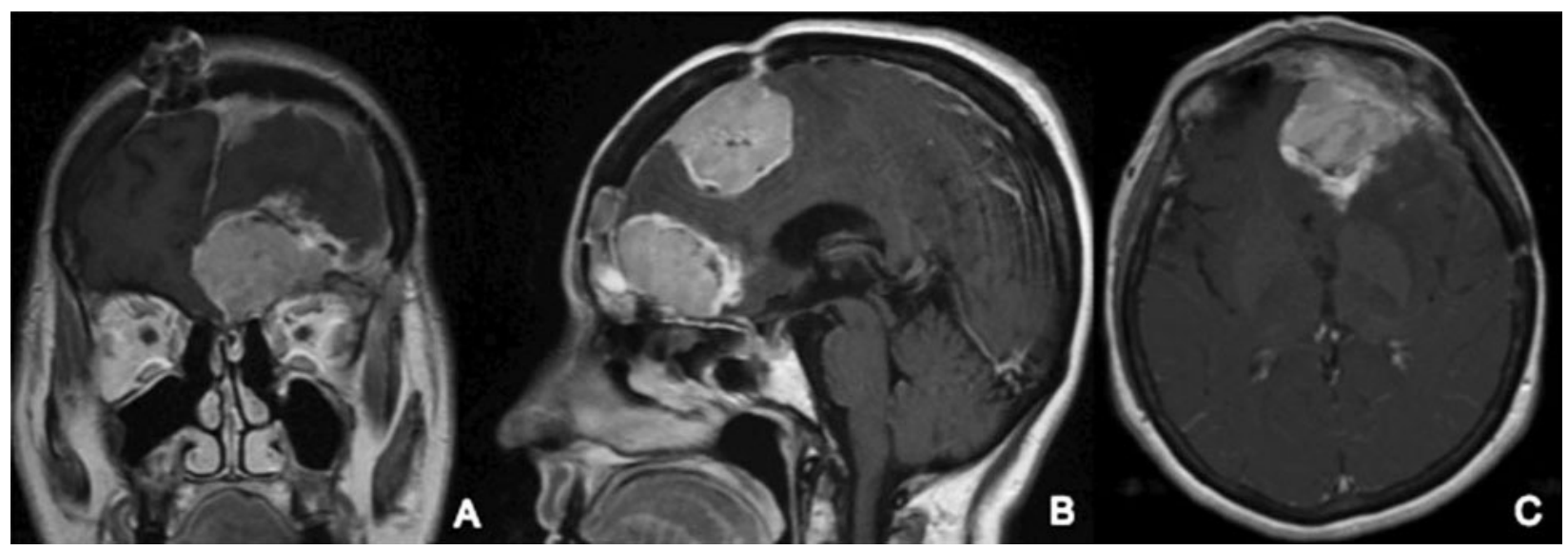

Figura 1. Meningioma anaplásico que compromete el planum esfenoidal y falcino izquierdo. Secuencias coronal (A), sagital (B) y axial (C) de T1 con gadolinio.

reconocimiento meningotelial, con necrosis, nucléolos grandes, incremento del número de mitosis (hasta 19 por 10 (GA), hialinización focal y hemorragia reciente con reactividad para EMA y en forma prominente para los receptores de progestágenos; el Ki67 se calculó en un $15 \%$. En conjunto, los hallazgos del tumor esfenoidal y frontal fueron compatibles con un meningioma anaplásico.

Por la imposibilidad para llevar a cabo una resección completa y por la invasión del parénquima cerebral y las características histológicas, se decidió efectuar radiocirugía estereotáxica fraccionada, seguida de hidroxiurea (1.000 mg c/día), que se suspendió 63 días después, al encontrar crecimiento tumoral. Posteriormente, se inició imatinib sin respuesta ante el compromiso de la región orbitaria. En ese momento, se practicó una tercera resección quirúrgica parcial, seguida de everolimus (10 mg c/día) más octreotide LAR (30 mg IM c/28 días), alcanzando respuesta parcial. Luego de nueve meses de esta intervención, la paciente tiene un óptimo estado clínico y un adecuado control imaginológico de la enfermedad (figura 2).

\section{Discusión}

Desde nuestro conocimiento, el presente caso constituye uno de los primeros reportes que demuestra la eficacia del uso de un inhibidor mTOR en combinación con octreotide LAR en un paciente con meningioma anaplásico refractario a otras intervenciones, incluyendo la radioterapia.

Los inhibidores del complejo mTOR modulan el crecimiento, la movilidad, la proliferación y la sobrevida de las células tumorales, incluyendo aquellas derivadas del meningotelio (figura 3). Recientemente, Barlier y colaboradores desarrollaron un modelo in vitro que demostró una elevada respuesta (mayor del $40 \%$ ) de células derivadas de meningioma anaplásico dependientes de la activación de mTOR AKT después de la administración de everolimus más octreotide o pasireotide ${ }^{10}$. En adición, Pachow demostró en xenoinjertos ortotópicos

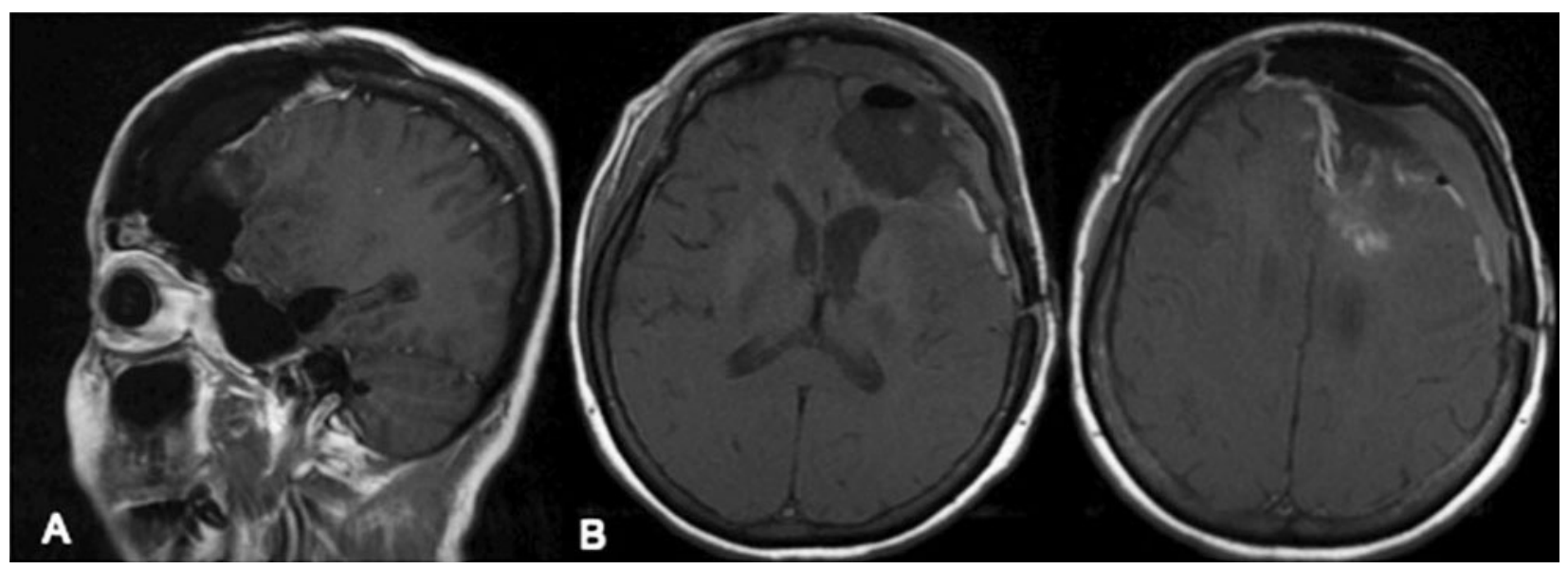

Figura 2. Imagen por RM secuencias sagital (A) y axial (B) en T1 con gadolinio que demuestra respuesta de la enfermedad después de nueve meses de tratamiento con everolimus y octreotide. 


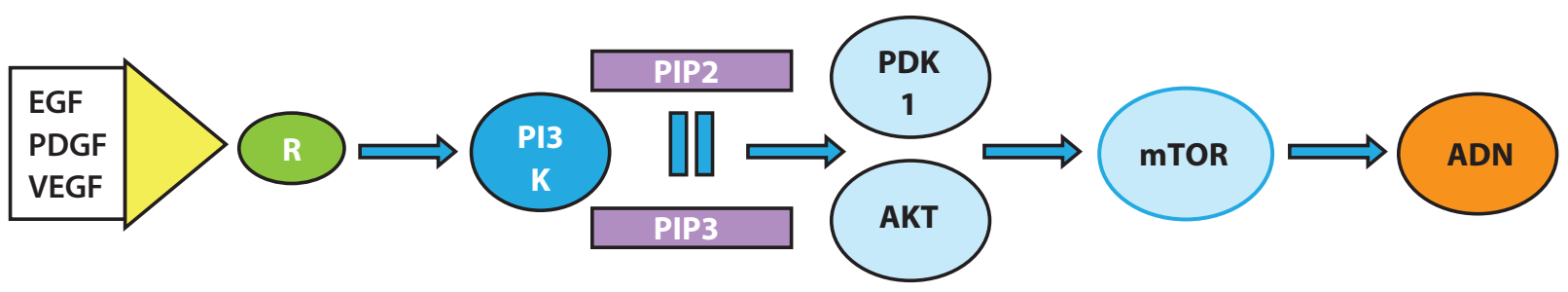

Figura 3. La inhibición del complejo mTOR influye de manera directa en la proliferación, angiogénesis y supervivencia celular, modulando la reparación y replicación del ADN en las células meningoteliales.

que el temsirolimus reduce el crecimiento de las células anaplásicas del meningioma en un $70 \%$, evento que se acompaña de la disminución en el índice mitótico cuantificado por el Ki 67 y por el descenso en la actividad de mTOR 1 medido por la fosforilación de P70S6K ${ }^{11}$.

La somatostatina es un neuropéptido producido en el hipotálamo con una reconocida actividad antitumoral en las neoplasias neuroendocrinas, donde actúa sobre la angiogénesis y la proliferación vía inducción de la apoptosis por la caspasa $3^{12}$. El octreotide es una molécula sintética análoga de la somatostatina, con una alta afinidad por sus receptores, en especial por el tipo 2 (RS2). Su beneficio potencial en los pacientes con meningiomas de alta gradación equipara su baja toxicidad ${ }^{7}$. Un estudio previo demostró que el 84 y 100\% de los meningiomas atípicos y anaplásicos presentan una alta expresión de RS2, hallazgo que se relaciona con una elevada inmunorreactividad para CD105, CD31 y Ki 67. Estos hallazgos permitieron generar la hipótesis de que la ganancia de RS2 en los meningiomas de alto grado promueve la neoangiogénesis y la proliferación celular anómala ${ }^{7}$. Según Durand y colaboradores, la elevada expresión de RS2 en los meningiomas de alto grado esporádicos sugiere una tumorigénesis independiente a NF2 que podría ser modulada, al menos en parte, por el receptor para el factor de crecimiento epidérmico (EGFR) y el Her2 ${ }^{13}$.

Para probar la eficacia de los análogos de somatostatina en pacientes con meningiomas de alto grado recurrentes, Jhonson desarrolló un experimento clínico fase 2 usando el análogo de corta duración $(500$ mcg c/8 horas) hasta intolerancia o progresión. El estudio incluyó ocho pacientes con meningiomas de alto grado sin mayor respuesta y con una mediana de SG tras el inicio de la medicación de 2,7 años. La expresión de RS2 no se asoció con el período libre de progresión y dos sujetos experimentaron un período de estabilidad superior a los 10 años? $^{7}$ En el momento, se está desarrollando un estudio multicéntrico (NCT00813592) para probar la eficacia y seguridad del pasireotide (SOM230) en pacientes con meningiomas agresivos recurrentes.

Por otra parte, los avances tecnológicos traen consigo día a día nuevas opciones terapéuticas dirigidas contra diversos blancos moleculares. Dentro del abanico de posibilidades, se encuentra el interferón alfa-2B, que en estudios in vitro ha demostrado la inhibición del crecimiento de las células meningoteliales anormales, permitiendo la estabilidad clínica en algunos $^{14-16}$. Otras opciones de manejo son los inhibidores de los receptores de estrógenos, progestágenos y andrógenos, gracias a la sobreexpresión nuclear de algunos de estos elementos en los meningiomas ${ }^{8}$. Otras alternativas podrían ser los inhibidores de los receptores de la hormona de crecimiento, como el pegvisomant, que se encuentra en desarrollo preclínico ${ }^{17,18}$. Entre las moléculas más estudiadas, está el bevacizumab, un antiangiogénico con el que se ha obtenido un adecuado control de la patología a largo plazo en breves series exploratorias.

De igual forma, los inhibidores reversibles del EGF (gefitinib, erlotinib), los inhibidores del TGF beta/SMAD y de la vía PLC-C1-PKC, que son claves en la proliferación celular de los meningionas agresivos ${ }^{8}$.

\section{Conclusiones}

Los meningiomas de alto grado son tumores de difícil control a pesar de la cirugía y del uso de la radioterapia y quimioterapia convencional. Sin embargo, en el último quinquenio se ha abierto un sinnúmero de posibilidades terapéuticas en desarrollo, muchas de ellas favorecidas por informes recientes sobre el genotipo completo de estas neoplasias. Nuestro caso constituye la primera evidencia clínica de la utilidad del everolimus más el octreotide LAR en una mujer con un meningioma anaplásico recurrente. 


\section{Referencias}

1. Modha A, Gutin PH. Diagnosis and treatment of atypical and anaplastic meningiomas: a review. Neurosurgery. 2005:57(3):538-50

2. Hanft $S$, Canoll $P$, Bruce JN. A review of malignant meningiomas: diagnosis, characteristics, and treatment. J Neurooncol. 2010;99(3):433-43

3. Norden AD, Drappatz J, Wen PY. Targeted drug therapy for meningiomas. Neurosurg Focus. 2007;23(4):E12.

4. Schulz C, Mathieu R, Kunz U, Mauer UM. Treatment of unresectable skull base meningiomas with somatostatin analogs. Neurosurg Focus. 2011;30(5):E11.

5. Brastianos PK, Horowitz PM, Santagata S, Jones RT, McKenna $A$, Getz $G$, et al. Genomic sequencing of meningiomas identifies oncogenic SMO and AKT1 mutations. Nat Genet. 2013:45(3):285-9.

6. Clark VE, Erson-Omay EZ, Serin A, Yin J, Cotney J, Ozduman K, et al. Genomic analysis of non-NF2 meningiomas reveals mutations in TRAF7, KLF4, AKT1 and SMO. Science. 2013;339(6123):1077-80.

7. Jhonson MD, O'Conell M, Vito F, Bacos RS. Increased STAT-3 and synchronous activation of Raf-1-MEK-1-MAPK, and phosphatidylinositol 3-Kinase-Akt-mTOR pathways in atypical and anaplastic meningiomas. J Neurooncol. 2009;92(2):129-36.

8. Wen PY, Quant E, Drappatz J, Beroukhim R, Norden AD. Medical therapies for meningiomas. J Neurooncol. 2010;99(3):365-78.

9. Johnson DR, Kimmel DW, Burch PA, Cascino TL, Giannini C, Wu W, et al. Phase II study of subcutaneous octreotide in adults with recurrent or progressive meningioma and meningeal hemangiopericytoma. Neuro Oncol. 2011;13(5):530-5.

10. Barlier A, Graillon T, Defilles C, Mohamend A, Saveanu A. 419PD STRONG Additive effect of everolimus and octreotide or pasireotide on meningioma cells in vitro: a new therapeutic strategy for these tumors. Ann Oncol. 2012;23(suppl 9):ix144-51.

11. Pachow D, Andrae N, Kliese N, Angenstein F, Stork $O$, Wilisch-Neumann A, et al. mTORC1 inhibitors suppress meningioma growth in mouse models. Clin Cancer Res. 2013;19(5):1180-9.

12. Lattuada D, Casnici C, Venuto A, Marelli O. The apoptotic effect of somatostatin analogue SMS 201-995 on human lymphocytes. J Neuroimmunol. 2002;133(1-2):211-6.

13. Durand A, Champier J, Jouvet A, Labrousse F, Honnorat J, Guyotat J, et al. Expression of c-Myc, neurofibromatosis Type 2, somatostatin receptor 2 and erb-B2 in human meningiomas: relation to grades or histotypes. Clin Neuropathol. 2008:27(5):334-45

14. Lamar Z, Lesser GJ. Management of meningeal neoplasms: meningiomas and hemangiopericytomas. Curr Treat Options Oncol. 2011;12(3):230-9.

15. Koper JW, Zwarthoff EC, Hagemeijer A, Braakman R, Avezaat $C J$, Bergström $M$, et al. Inhibition of the growth of cultured human meningioma cells by recombinant interferon-alpha. Eur J Cancer. 1991;27(4):416-9.

16. McCutcheon IE, Flyvbjerg A, Hill H, Li J, Bennett WF, Scarlett $\mathrm{JA}$, et al. Antitumor activity of the growth hormone receptor antagonist pegvisomant against human meningiomas in nude mice. J Neurosurg. 2001;94(3):487-92.

17. Drake WM, Grossman AB, Hutson RK. Effect of treatment with pegvisomant on meningioma growth in vivo. Eur J Endocrinol. 2005;152(1):161-2.

18. Arena $S$, Barbieri F, Thellung S, Pirani P, Corsaro A, Villa V, et al Expression of somatostatin receptor mRNA in human meningiomas and their implication in in vitro antiproliferative activity. J Neurooncol. 2004;66(1-2):155-66.

19. Shih K, Rosenblatt P, Chowdhary SA, Weir A, Shepard G, Shastry $M$, et al. Combination of bevacizumab and everolimus in patients with refractory, progressive intracranial meningioma: a phase II trial of the Sarah cannon research institute. Neurooncology. 2012;14(suppl 6):vi101-5.

20. Chamberlain MC, Glantz MJ, Fadul CE. Recurrent meningioma: salvage therapy with long-acting somatostatin analogue. Neurology. 2007;69(10):969-73.

21. Klutmann S, Bohuslavizki KH, Brenner W, Behnke A, Tietje N, Kröger $S$, et al. Somatostatin receptor scintigraphy in postsurgical follow-up examinations of meningioma. J Nucl Med. 1998;39(11):1913-7.

22. De Jong $M$, Valkema $R$, Jamar $F$, Kvols LK, Kwekkeboom DJ, Breeman WA, et al. Somatostatin receptor-targeted radionuclide therapy of tumors: preclinical and clinical findings. Semin Nucl Med. 2002;32(2):133-40

23. Hanakita S, Koga T, Igaki H, Murakami N, Oya S, Shin M, et al. Role of Gamma Knife surgery for intracranial atypical (WHO Grade II) meningiomas. J Neurosurg. 2013;119(6):1410-4.

24. Hou J, Kshettry VR, Selman WR, Bambakidis NC. Peritumora brain edema in intracranial meningiomas: the emergence of vascular endothelial growth factor-directed therapy. Neurosurg Focus. 2013;35(6):E2

25. Wang S, Yang W, Deng J, Zhang J, Ma F, Wang J. Reduction in the recurrence of meningiomas by combining somatostatin receptor scintigraphy of (99m)Tc-HYNIC-octreotide SPECT/CT and radio guidance with a hand-held g-probe during surgery. Nucl Med Commun. 2013;34(3):249-53.

26. Bernal L, Cardona AF, Useche N, Bartels C, Bermúdez S, Jiménez $E$, et al. Terapia dirigida en meningiomas: genómica con nuevas perspectivas. RedLANO e-Journal 2013.

27. Moazzam AA, Wagle N, Zada G. Recent developments in chemotherapy for meningiomas: a review. Neurosurg Focus. 2013;35 (6):E18.

28. Muhr C, Gudjonsson O, Lilja A, Hartman M, Zhang ZJ, Langstrom B. Meningioma treated with interferon-alpha, evaluated

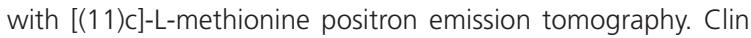
Cancer Res. 2001;7(8):2269-76. 